\title{
Towards the development of a MEMS-based health monitoring system for lightweight structures
}

\author{
Francesco Caimmi $^{1, *}$, Matteo Bruggi ${ }^{2}$, Stefano Mariani ${ }^{2}$, Paolo Bendiscioli ${ }^{3}$ \\ 1 Politecnico di Milano, Dipartimento di Chimica, Materiali e Ingegneria Chimica "Giulio Natta", \\ Piazza L. da Vinci 32, 20133 Milano, Italy; E-Mail: francesco.caimmi@polimi.it \\ 2 \\ Politecnico di Milano, Dipartimento di Ingegneria Civile e Ambientale, Piazza L. da Vinci 32, \\ 20133 Milano, Italy; E-Mails: matteo.bruggi@polimi.it (M.B.); stefano.mariani@polimi.it (S.M.) \\ 3 STMicroelectronics, AMS Product Division, Via Tolomeo 1, 20010 Cornaredo, Italy; E-Mail: \\ paolo.bendiscioli@st.com
}

* Author to whom correspondence should be addressed; E-Mail: francesco.caimmi@polimi.it (F.C.); Tel.: +39-02-2399-4711; Fax: +39-02-7063-8173.

Published: 1 June 2014

\begin{abstract}
We recently proposed a surface-mounted structural health monitoring (SHM) scheme based on commercial, low-cost inertial MEMS sensors. While such commercialoff-the-shelf sensors are not very accurate, their low cost and negligible weight allow them to be deployed in dense arrays, possibly overcoming inaccuracy through redundancy. Taking the sensor characteristics into account, the development of a MEMS-based SHM method for lightweight structures like thin plates, is tackled from two different viewpoints: sensor accuracy verification, and optimal sensor placement. To assess the accuracy, a preliminary investigation was run on standard composite specimens for delamination testing, adopting a single MEMS three-axis accelerometer. A theoretical interpretation of the results, based on beam bending theory, showed the ability of the system to provide a one-to-one relationship between the delamination length and the sensor output. Concerning the placement of the sensors, an approach for their optimal deployment was developed, using a topology optimization-like formulation. Such formulation looks for the optimal layout of the network by maximizing the overall sensitivity of the output to a damage possibly located anywhere. In this work, accounting for the characteristic sizes of a structural element and of the MEMS package, which might differ by orders of magnitude, we introduce a multi-scale (actually, two-scale) approach to sensor deployment. It is shown that, no matter what the location and size of the damaged area are, a trivial array of evenly spaced sensors does not represent the optimal solution for SHM.
\end{abstract}

Keywords: Structural Health Monitoring; composite laminates; inertial MEMS. 


\section{Introduction}

Health monitoring of real-life structures, especially safety-critical ones, is nowadays perceived as a paramount field of study [1]. Structural health monitoring (SHM) systems must be able to provide timely information during the whole life cycle of the structures themselves [2]. In order to prevent failures under service or extreme external actions, a reliable model of the structure should be continuously fed by the information collected by permanently installed sensors.

With the aim to develop a SHM system for lightweight composites structures like those found in aerospace or civil engineering fields, the authors started to develop an approach based on commercial, low-cost inertial MEMS sensors [3-5]. Such sensors, which are very lightweight, look appropriate for composites laminates; to monitor these structures, they can be deployed on the outer surface and avoid the drawbacks associated with the embedding of the devices inside the material [5]. Although MEMS sensors are typically not very accurate, their low cost and negligible weight allow a deployment in dense arrays, possibly overcoming inaccuracy through redundancy.

There are many issues to be studied for the development of appropriate SHM systems for composites. In this work, the attention is focused on two of them: first, the ability of the sensors to provide data useful for SHM purposes is assessed, by adopting simple schemes for standard laboratory samples (Section 2); second, a sensor placement strategy is addressed, with a method based on topology optimization (Section 3).

\section{SHM accuracy assessment}

Building on the work done in [5] to evaluate the possibility of using a MEMS-based SHM scheme for composite laminates, a simple approach has been adopted for two standard specimens commonly used for delamination resistance testing: the double cantilever beam (DCB) and the end loaded split (ELS) ones. Schemes of the deformed shape of the two specimens, and of the relevant boundary conditions are given in Fig. 1. Both the setups are typically used to test very slender (beam like) composite samples with rectangular cross section, containing a delamination artificially inserted on the laminate mid-plane during manufacturing; the initial delamination length in the tests was $a=60 \mathrm{~mm}$. The samples were connected to a dynamometer through the load-blocks depicted as well in Fig. 1, and point forces were applied; Fig. 1(c) shows a loaded DCB sample.

Samples made of a carbon fabric reinforced epoxy, were instrumented with a single LIS3LV02DQ three-axis, digital output MEMS accelerometer [6]. As the device is sensitive to the gravity acceleration too, its tilting relative to the vertical direction can be easily obtained. An evaluation board, carrying the accelerometer, a microprocessor for data acquisition and a USB port for connection with a PC, was mounted on the samples using a back plate as in Fig. 1(c); the sensor positioning for the two tests is shown in Figs. 1 (a) and (b).

The test protocol adopted for both the setups has been the same as in [5]: on each specimen, a series of different load steps have been applied by prescribing a sinusoidal variation of the load point displacement $\delta$ with frequency $f_{\delta}=0.5 \mathrm{~Hz}$. Within each step, the baseline displacement $\delta_{0}$ has been progressively increased to stably advance the delamination front only at the beginning of the loading. 
Figure 1. Schemes of the deformed shape (a) of the DCB specimen, and (b) of the ELS specimen; (c) a DCB specimen under load.

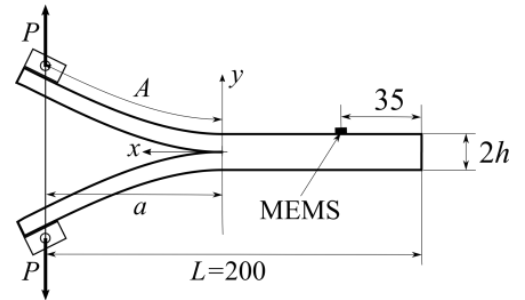

(a)

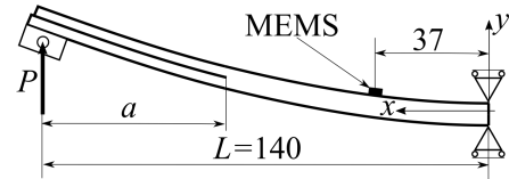

(b)

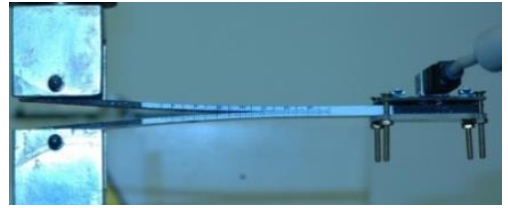

(c)

Figure 2. (a) DCB test: amplitude of the peak of the Fourier transform of the acceleration at $f_{\delta}$, as a function of the delamination length; (b) ELS test: normalized rotation at the MEMS position, as a function of the delamination length.

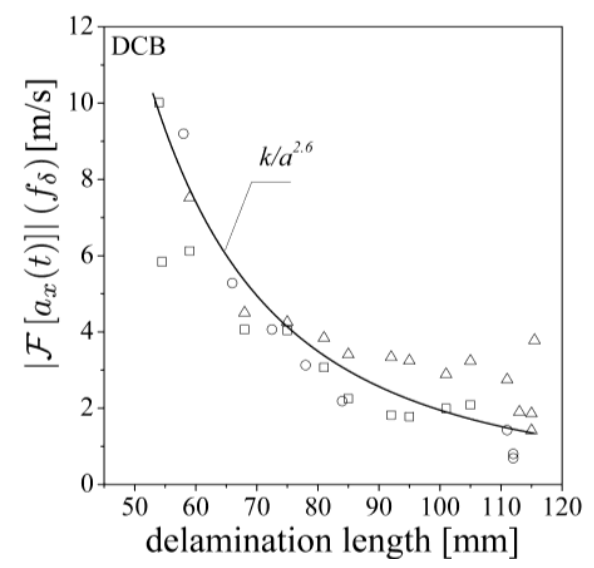

(a)

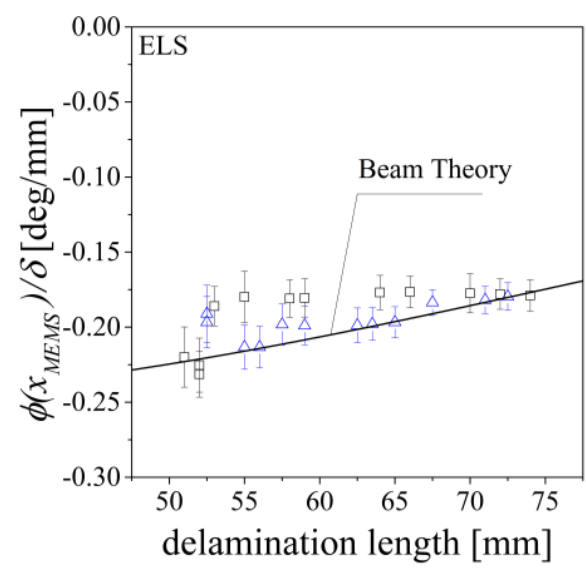

(b)

Different monitoring schemes have been adopted for the two setups. For the DCB test, a semiempirical correlation was chosen, see [5], handling the acceleration $a_{x}$ measured along the horizontal axis rather than that along the vertical one, which was instead used in [5]. As the raw signal was too noisy to suit the purpose, its Fourier transform $F\left[a_{x}(t)\right]$ was used: the value of the peak of the transform at the forcing frequency $f_{\delta}$ showed a good correlation with the delamination length, as reported in Fig. 2(a). The black line shows the data best fit obtained with the aforementioned semiempirical correlation.

As for the ELS test, the rotation of the sensor with respect to the horizontal axis was measured. The resulting rotations are compared in Fig. 2(b) with those still predicted by beam bending, showing again a very good agreement.

\section{MEMS placement strategy}

As briefly reported above, MEMS accelerometers can be effectively used to detect local rotations of the mid-surface of thin plates and, therefore, also changes induced by damage events. 
To provide a numerical framework able to optimize the topology of a sensors network, we assume the plate to be space-discretized into finite elements (FEs), characterized by a size $h$. Following [7], a formulation is discussed to deploy a maximum number $\bar{N}$ of sensors so as to maximize an objective function defining the sensitivity to the damage. To build such objective function, the plate response to the external loads is computed in terms of nodal rotations in the undamaged and damaged cases. The two solutions are then post-processed, in order to compute vectors of element-wise rotations $\widehat{\boldsymbol{\vartheta}}_{i}$ in the undamaged state and $\boldsymbol{\vartheta}_{k i}$ in the damaged states, where subscript $i=1, \ldots, n$ is an index running over the elements of the mesh and subscript $k=1, \ldots, n$ is an additional index running over all the possible damage locations. The norm $\left\|\boldsymbol{\vartheta}_{k i}-\widehat{\boldsymbol{\vartheta}}_{i}\right\|$ thus accounts for the overall structural changes induced by damage.

If the element-wise variable $0 \leq x_{i} \leq 1$ represents an ad-hoc discrete density field, it can account for the presence of the sensor over the $i$-th element. The adopted strategy for the distribution of sensors over the plate consists in maximizing the sum $\sum_{i=1}^{n} x_{i}^{p}\left\|\boldsymbol{\vartheta}_{i}-\widehat{\boldsymbol{\vartheta}}_{i}\right\|$, constrained by the maximum allowed number of sensors according to $\sum_{i=1}^{n} x_{i} \leq \bar{N}$; the tuning exponent $p \geq 1$ allows steering the solution towards a pure 0-1 discrete distribution. As already reported in [4,7], to balance the effects of all the possible damage locations, a weighted objective function and relevant optimization problem are defined as:

$$
\max _{0 \leq x_{i} \leq 1} \sum_{k=1}^{n}\left[\frac{\sum_{i=1}^{n} x_{i}^{p}\left\|\boldsymbol{\vartheta}_{k i}-\widehat{\boldsymbol{\vartheta}}_{i}\right\|}{\max _{i} x_{i}^{p}\left\|\boldsymbol{\vartheta}_{k i}-\widehat{\boldsymbol{\vartheta}}_{i}\right\|}\right] \text { s.t. } \sum_{i=1}^{n} x_{i} \leq \bar{N}
$$

An application of this formulation is reported for a thin plate (side length $L$ to thickness $t$ ratio on the order of 1,000), clamped along its whole boundary and subject to a distributed out-of-plane load. $\bar{N}=8$ sensors, with size $l=L / 400$, are assumed to be optimally placed to detect a damage of size $s=L / 20$, possibly located anywhere over the plate. Such ratios have been considered to allow for real-life situations, when structures have dimensions on the order of meters, damaged areas extending over parts of the structures before any possible failure event have dimensions on the order of tens of centimeters at most, and packaged MEMS sensors have a size of a few millimeters.

A single-scale implementation of the formulation in Eq. (1) would lead to an optimization problem with 160,000 unknowns $x_{i}$, and require the evaluation of 401 vectors of element-wise rotations $\widehat{\boldsymbol{\vartheta}}_{i}$ and $\boldsymbol{\vartheta}_{k i}$, both with 160,000 components. To avoid this computational burden, a two-scale procedure is here adopted. The optimization performed at the macro-scale is exploited to define eight regions (allowing for the problem symmetries) with characteristic size $h=s=L / 20$, where sensors should be placed, see Figs. 3(a) and 3(b). A series of micro-scale analyses is adopted to perform the optimization for each one of the above identified regions, allowing for a characteristic size $h=l=L / 400$ of the sensors, see Figs. 3(c) and 3(d) which show the outcomes relevant to the region highlighted in Fig. 3(b). Standard sub-modelling techniques have been adopted to enforce suitable boundary conditions to the micro-scale analyses. Results at the micro-scale concerning the optimal placement in the other micro-scale regions are herein omitted for the sake of brevity, as they can be simply derived from Fig. 3(d) by exploiting symmetry. The outlined two-scale approach provides a remarkable save in terms of computational time, since only two optimizations involving 400 unknowns $x_{i}$ are to be performed. 
Figure 3. Sensor network topology for a clamped square plate, with damage possibly located anywhere. (left column) Non-dimensional objective function; (right column): optimal sensor deployment; (top row): macro-scale results, $\bar{N}=8$; (bottom row): microscale results, $\bar{N}=1$.

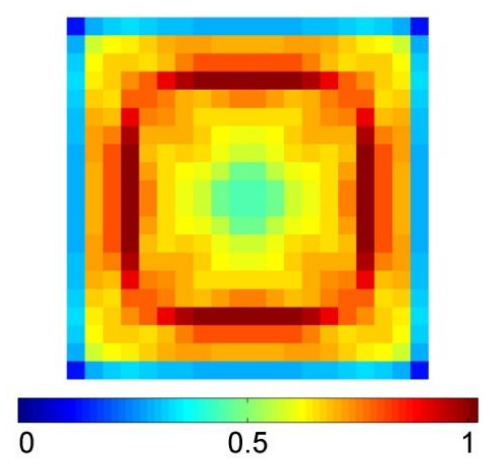

(a)

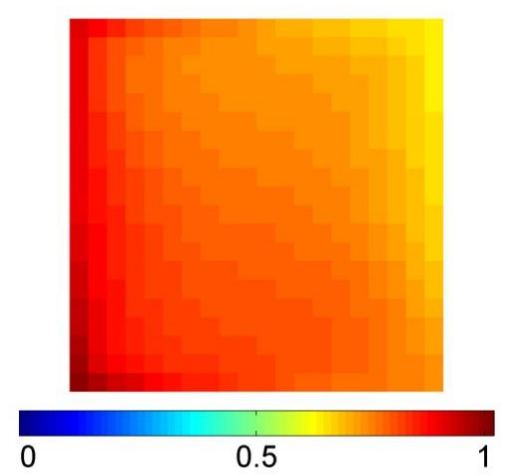

(c)

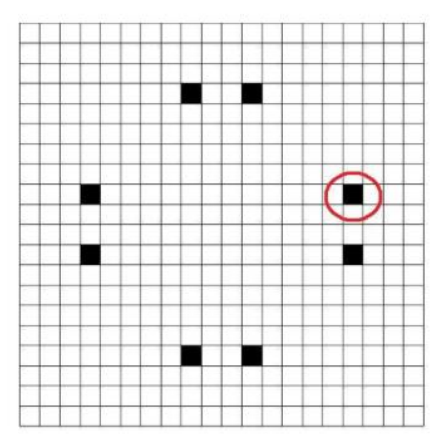

(b)

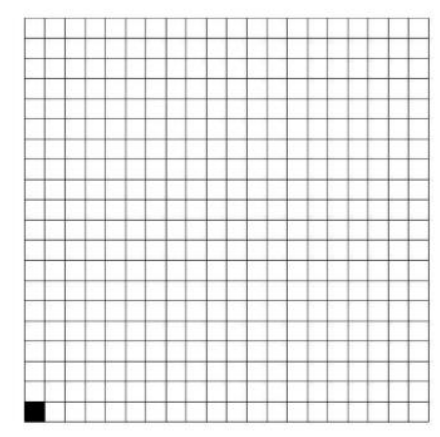

(d)

\section{Conclusions}

From the two viewpoints explored in this work, the proposed MEMS-based SHM scheme looks promising. The preliminary analysis of very simple beam like geometries showed that inertial MEMS sensors could be effectively exploited with minimal signal post-processing, taking advantage of structural models to detect delamination growth.

Concerning the sensor placement algorithm, optimization has been shown to be a valuable tool to define the topology of a sensor network over flexible, lightweight structures. The aim here considered has been the maximization of the sensitivity of the output of the SHM system to a damage possibly located anywhere. The two-scale approach, used to improve the computational performance of the previously developed algorithm, showed the potential for very large speed-ups without accuracy loss. This provides a preliminary rationale to cope with large-scale problems found in practical applications, which often involve remarkable differences in terms of size of the structures, sensors and expected damages. 


\section{Acknowledgments}

A partial financial support from Fondazione Cariplo through project Safer Helmets is gratefully acknowledged.

\section{References}

1. Achenbach, J. D. Structural health monitoring - What is the prescription?. Mechanics Research Communications 2009, 36, 137-142.

2. Mukhopadhyay, S. C.; Chase, J. G.; Meyendorf, N. Editorial Special Issue on Sensors Systems for Structural Health Monitoring. Sensors Journal, IEEE 2009, 9, 1319-1321.

3. Mariani, S.; Corigliano, A.; Caimmi, F.; Bruggi, M.; Bendiscioli, P.; De Fazio, M. Health monitoring of flexible composite plates: A MEMS-based approach. MicroTech Conference \& Expo 2012, Santa Clara, CA (USA), 18-21 June 2012, pp 227-230.

4. Mariani, S.; Bruggi, M.; Caimmi, F.; Bendiscioli, P.; De Fazio, M. Sensor deployment over damage-containing plates: A topology optimization approach. Journal of Intelligent Material Systems and Structures 2013, 24, 1105-1122.

5. Mariani, S.; Corigliano, A.; Caimmi, F.; Bruggi, M.; Bendiscioli, P.; Fazio, M. D. MEMS-based surface mounted health monitoring system for composite laminates. Microelectronics Journal 2013, 44, 598-605.

6. STMicroelectronics. LIS3LV02DQ Datasheet, 2005.

7. Bruggi, M.; Mariani, S. Optmization of sensor placement to detect damage in flexible plates. Engineering Optimization 2013, 45, 659-676.

(C) 2014 by the authors; licensee MDPI, Basel, Switzerland. This article is an open access article distributed under the terms and conditions of the Creative Commons Attribution license (http://creativecommons.org/licenses/by/3.0/). 